\title{
Depth-Based Shape-Analysis
}

\author{
Yi Hong ${ }^{1}$, Yi Gao ${ }^{3}$, Marc Niethammer ${ }^{1,2}$, and Sylvain Bouix ${ }^{4}$ \\ 1 University of North Carolina (UNC) at Chapel Hill, NC, USA \\ 2 Biomedical Research Imaging Center, UNC-Chapel Hill, NC, USA \\ ${ }^{3}$ University of Alabama at Birmingham, Birmingham, AL, USA \\ ${ }^{4}$ Psychiatry Neuroimaging Laboratory, Brigham and Women's Hospital, \\ Harvard Medical School, Boston, MA, USA
}

\begin{abstract}
In this paper we propose a new method for shape analysis based on the depth-ordering of shapes. We use this depth-ordering to non-parametrically define depth with respect to a normal control population. This allows us to quantify differences with respect to "normality". We combine this approach with a permutation test allowing it to test for localized shape differences. The method is evaluated on a synthetically generated striatum dataset as well as on a real caudate dataset.
\end{abstract}

\section{Introduction}

Population-based shape analysis is of high importance to discriminate for example normal subjects from subjects with a particular disease. Many methods for shape analysis exist. They can be subdivided into global and local analysis methods. Global analysis methods are designed to detect whether population shape differences exist [5], but cannot generally locate where these shape differences may be, which limits their ability to provide intuitive insights into the underlying biological mechanism. The main attraction of such methods is that they often avoid establishing dense correspondences between shapes through registration. In contrast, while point-to-point correspondences between shapes allow precise local shape analysis, establishing these correspondences is one of the main sources of inaccuracy as any misregistration may create artifacts with respect to the final shape analysis results. Nevertheless, a variety of methods for local shape analysis have been proposed and successfully used [8]71]. In this work, we explore an alternative method that allows for localized shape analysis, but only needs very limited (e.g., rigid or affine) spatial alignment of shapes. Our method uses a depth-ordering of shapes to allow to compare shape populations.

\section{Our Main Contributions in This Paper Are:}

1) We propose using depth-ordering on shapes for statistical shape analysis.

2) We develop an algorithm for the fast computation of band-depth for shapes represented through binary indicator functions.

3) We define statistical tests to differentiate shape populations globally and locally without an explicit computation of dense correspondences.

4) We demonstrate the method on synthetic and real datasets.

P. Golland et al. (Eds.): MICCAI 2014, Part III, LNCS 8675, pp. 17-24, 2014.

(C) Springer International Publishing Switzerland 2014 
Sec. 2 describes how to depth-order shapes and discusses how to compute such a depth-ordering fast. Sec. 3 proposes statistical approaches using depth-ordering for shape analysis. In Sec. 4, we present experimental results for synthetic and real datasets. Sec. 5 concludes the paper with a summary.

\section{Depth-Ordering of Shapes}

A challenge in shape analysis is that there is no canonical ordering of shapes. Here we leverage the work on ordering of functions from the statistics literature and extend it to shapes [6. Once defined the ordering can be used to generalize traditional order statistics, such as the median or the inter-quartile range, to shape ensembles. Recently, band-depth has been proposed as one possible way for ordering functions 9]. Intuitively, the deeper a function is buried within a dataset the more central it is. The deepest function corresponds to the within-sample median function. Band-depth has been used to define a functional boxplot 9 . It has also been extended to contour boxplots [11] defining band-depth on contours for the visualization of ensemble data. What makes band-depth attractive for shape-ordering is that shapes can be analyzed as functions if they are represented by indicator functions, i.e., by binary functions that are 1 inside and 0 outside of a shape [3]. Band-depth for binary shape representations relates to set unions and intersections, and it is a natural functional representation of shape.

Given a set of shapes as binary functions, $\left\{\mathrm{y}_{1}, \mathrm{y}_{2}, \ldots, \mathrm{y}_{n}\right\}$, with dimension of $\left(s_{x}, s_{y}, s_{z}\right)$, we vectorize them and obtain binary vectors $\mathrm{y}_{i} \in\{0,1\}^{p}$, where $p=s_{x} \times s_{y} \times s_{z}$. The band-depth for each shape $\mathrm{y}$ is defined as follows:

$$
B D_{n}^{(j)}(\mathrm{y})=\frac{1}{C} \sum_{1 \leq i_{1}<i_{2}<\cdots<i_{j} \leq n} I\left\{G(\mathrm{y}) \subseteq B\left(\mathrm{y}_{i_{1}}, \cdots, \mathrm{y}_{i_{j}}\right)\right\} .
$$

Here, $1 \leq j \leq J$, and $J$ is the number of observations used for defining the band, $C$ is a normalization constant equal to the number of admissible permutations. $G(\mathrm{y})$ is the graph of the function, $G(\mathrm{y})=\{(\mathbf{x}, \mathrm{y}(\mathbf{x})): \mathbf{x} \in \mathbb{I}\} . B$ is the band delimited by the observations given as its arguments. That is, $B\left(\mathrm{y}_{i_{1}}, \cdots, \mathrm{y}_{i_{j}}\right)=$ $\left\{(\mathbf{x}, \mathrm{y}(\mathbf{x})): \mathbf{x} \in \mathbb{I}, \min _{r=i_{1}, \cdots, i_{j}} \mathrm{y}_{r}(\mathbf{x}) \leq \mathrm{y}(\mathbf{x}) \leq \max _{r=i_{1}, \cdots, i_{j}} \mathrm{y}_{r}(\mathbf{x})\right\} . I\{$.$\} de-$ notes the indicator function, which evaluates to 1 if the graph of the function is within the band, or to 0 , otherwise. Since the band depth on binary functions may result in many ties for the resulting depth, it can be modified [9] to

$$
M B D_{n}^{(j)}(\mathrm{y})=\frac{1}{C} \sum_{1 \leq i_{1}<i_{2}<\ldots<i_{j} \leq n} \lambda_{m}\left\{A\left(\mathrm{y} ; \mathrm{y}_{i_{1}}, \ldots, \mathrm{y}_{i_{j}}\right)\right\}
$$

where $A_{j}(\mathrm{y}) \equiv A\left(\mathrm{y} ; \mathrm{y}_{i_{1}}, \ldots, \mathrm{y}_{i_{j}}\right)$ and $A_{j}(y) \equiv\left\{\mathbf{x} \in \mathbb{I}: \min _{r=i_{1}, \ldots, i_{j}} \mathrm{y}_{r}(\mathbf{x}) \leq\right.$

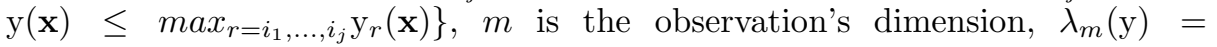
$\lambda\left(A_{j}(\mathrm{y})\right) / \lambda(\mathbb{I})$ and $\lambda$ is the Lebesgue measure on $\mathbb{R}^{m}$.

However, albeit its conceptual simplicity, one of the main limitations of the band-depth computation is its computational complexity. Therefore, recently a 
fast method to compute band-depth has been proposed 10 which is based on computing local curve ranks. However, the proposed algorithm is ill-suited for binary shape representations as it does not consider ranking ties for the modified band-depth (MBD) nor special cases where curves can change ordering without affecting the rank of a specific curve. For binary representations ranking can be avoided as at any point only two values are possible. The computation of MBD can then be accomplished efficiently for $J=2$. Our algorithm is as follows:

Step 0) Given $n$ binary volumes, $\left\{\mathrm{y}_{i}\right\}_{i=1}^{n}$, vectorize them: $\mathrm{y}_{i} \in\{0,1\}^{p}$.

Step 1) At each location, $k$, for a given value $v(k) \in\{0,1\}$, we count the number

of functions that have a value larger $\left(n_{a}\right)$, smaller $\left(n_{b}\right)$ or equal $\left(n_{t}\right)$ to $v$ :

- if $v(k)=0$, then $n_{a}=\sum_{i=1}^{n} \mathrm{y}_{i}(k), n_{b}=0$, and $n_{t}=n-n_{a}-1$

- if $v(k)=1$, then $n_{a}=0, n_{b}=\sum_{i=1}^{n}\left(1-\mathrm{y}_{i}(k)\right)$, and $n_{t}=n-n_{b}-1$

Step 2) We then calculate the number of pairwise combinations containing $v(k)$ :

$$
C_{k}(v(k))=n_{a} n_{b}+\left(n_{a}+n_{b}\right) n_{t}+n_{t}\left(n_{t}-1\right) / 2+\left(n_{a}+n_{b}+n_{t}\right) .
$$

For binary functions $n_{a}$ and $n_{b}$ cannot simultaneously be different from zero. Furthermore, $n_{a}+n_{b}+n_{t}=n-1$, which simplifies the expression to

$$
C_{k}(v(k))=\left(n_{a}+n_{b}\right) n_{t}+n_{t}\left(n_{t}-1\right) / 2+n-1 .
$$

Step 3) The modified band-depth for a curve $\mathrm{y}_{i}$ is then

$$
M B D\left(\mathrm{y}_{i}\right)=\frac{1}{p}\left(\begin{array}{l}
n \\
2
\end{array}\right)^{-1} \sum_{k=1}^{p} C_{k}\left(\mathrm{y}_{i}(k)\right),
$$

where the notation $C_{k}\left(\mathrm{y}_{i}(k)\right)$ denotes computing $C_{k}$ based on the coefficients $n_{a}, n_{b}, n_{t}$ given by the value of $\mathrm{y}_{i}$ at location $k$.

In comparison to the original band-depth algorithm our computing complexity is reduced from $O\left(p n^{3}\right)$ to $O(p n)$. This makes the computation for large populations and large multi-dimensional shapes (we will focus on $2 \mathrm{D}$ surfaces in 3D here) possible. Furthermore, it enables us to perform permutation tests based on band-depth computations as discussed in Sec. 3 .

\section{Statistics Using Depth-Ordering}

Band-depth measures the relationship between a shape and a reference population. A higher value indicates the shape is closer to the median, and a lower one indicates the shape is a potential outlier with respect to the reference population. Based on this property of band-depth we can perform global shape analysis as described in Sec. 3.1 as well as local shape analysis as described in Sec. 3.2. For all these analyses we assume that shapes have been pre-aligned as appropriate. Typically this will either involve a rigid, similarity or affine alignment of shapes to a template or some form of unbiased atlas-building method. The choice of transform will depend on the objective of a given study. E.g., if size differences should be included rigid alignment would be appropriate. The key ingredient to performing statistics using depth-ordering is to compute depth-ordering with respect to a reference population of shapes that are used as a non-parametric model of shapes, with respect to which depth is measured. 


\subsection{Global Shape Analysis}

Given a dataset $\left\{R_{i}\right\}$ containing a reference population of shapes, we compute the band-depth for a given datum $D$, from a set of input test shapes $\left\{D_{j}\right\}$, by computing the band-depth for all the data in $\left\{R_{i}\right\} \cup D$ and assign the resulting banddepth for $D$ to $D$, denoted as $B D\left(D ;\left\{R_{i}\right\}\right)$. This is sub-

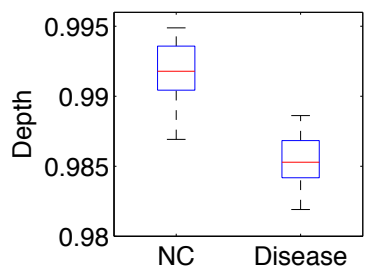

(a) w.r.t. ref.

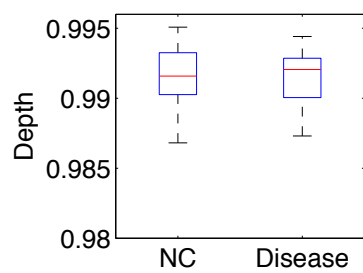

(b) without ref.

Fig. 1. Global shape analysis using band-depth with (a) and without (b) a reference population. A reference population allows detection of shape differences. stantially different from directly computing the band-depths for the dataset $\left\{D_{j}\right\}$. In the proposed method the reference population forms a "yard-stick" by which to judge data-depth. In the latter case data-depth is defined with respect to the dataset itself which is problematic as band-depth does not have a sense of directionality, but only a sense of how close a data-element is to the deepest data-element. For different populations which should be discriminated this consequentially leads to a data-mingling which no longer allows for a discrimination of the populations. To illustrate this effect Fig 1 shows results for the two approaches for the synthetic striatum data described in detail in Sec. 4.1. The proposed approach can clearly differentiate the populations whereas a joint computation of the band-depth is not successful.

\subsection{Local Shape Analysis}

The local analysis is based on the the central regions of the reference population. By gradually adding the deepest shapes according to their band-depth, one can assign $\alpha$ values, the proportion of the added reference shapes, describing the "centrality" of a shape population at each point in the domain. A test shape can be overlaid on this centrality map and the corresponding $\alpha$ values recorded on its surface, thus providing a local measure of shape abnormality. Fig. 2 illustrates this concept for a population of two-dimensional shapes. Given the reference shapes shown in Fig. 2(a), we compute their $\alpha$-central level sets based on the band-depth. As shown in Fig. 2(b), the deepest shape has the lowest $\alpha$ value (light blue) and the most outlying shape has the highest $\alpha$ value (dark blue). A local measure of "belonging" to the population can then be computed for a test shape by tracing the $\alpha$-central region it traverses as shown in Fig. 2(c). Note that some regions of the shape may not be covered by the reference shape population, so we use a dilation procedure starting from the boundary of $\alpha=1$ central region, evolving at a constant speed until all voxels of the volume are covered, e.g., the regions colored with the darkest blue in Fig. 2(c). 


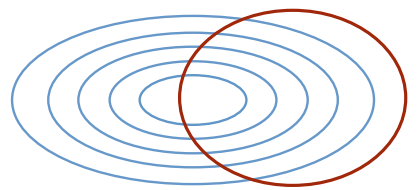

(a) Reference and test shapes

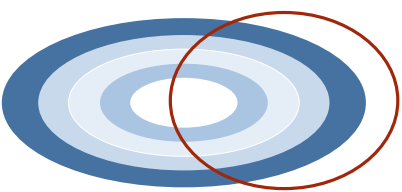

(b) $\alpha$-central region levelsets

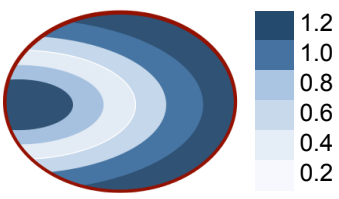

(c) Local measure of shape

Fig. 2. (a) Reference shape population (blue contours) defines (b) $\alpha$-central level sets (light to dark blue corresponds to most to least central) that provides a local measure (c) of how deeply a shape (red) is buried with respect to the reference population. The dilated region is colored with the darkest blue and has a value greater than 1 .

\section{Experimental Results}

\subsection{Synthetic Data Experiments}

Using synthetic data allows us to introduce a predefined shape change which we wish to recover using our proposed approach. We used the technique described in 2] to generate large data sets of realistic shapes with known deformations. In short, a manifold learning technique is used to generate arbitrarily many shapes from a small training sample. A joint clustering algorithm is then applied to parcellate each shape's surface into small regions which are consistently located across all shapes. Finally, a Log-Euclidean framework is used to introduce smooth, invertible and anatomically realistic deformations to one or multiple regions as defined by the clustering. For this application, we generated 160 shapes based on 27 manually traced striatums. We then modified 80 of them by thickening the putamen. We evenly divide 80 normal controls into two groups. One is used for the reference group (NC-Train), and the other is for testing (NC-Test). In the 80 abnormal subjects, we randomly pick 40 of them for testing.

Global Analysis. To test for group separability, we performed a permutation test (10000 permutations) on the mean depth of the NC-Test versus the abnormal group. When using the NC-Train to compute band depth, the resulting p-value is 0 , indicating the normal controls and the disease subjects are significantly different. On the other hand, as shown in Fig. 1, when pooling all shapes together to compute their band depths, no significant difference is detected.

Local Analysis. We used the NC-Train group to estimate the set of $\alpha$ central regions of a "normal" population and tested the median shapes of the NCTest and abnormal population against it as shown in Fig. 3)(a). In addition, to displaying $\alpha$ values, we performed a non parametric statistical analysis, based on a permutation test procedure. A template median shape is first computed, then $\alpha$ regions are computed for each group and differences in $\alpha$ values are recorded at each point of the template. 10000 permutations are performed and we count the number of $\alpha$ values that are larger than the one computed with no permutation. The p-values are shown in Fig. 3(b). Fig. 3)(c) reveals the false discovery rate (FDR) of p-values. 


\subsection{Real Data Experiments}

Magnetic Resonance Images (MRI) of the brains of 28 neuroleptic-naïve female subjects diagnosed with Schizotypal Personality Disorder (SPD) and of 25 female normal control subjects were acquired on a 1.5- $\mathrm{T}$ General Electric MR scanner. Spoiledgradient recalled acquisition (SPGR) images (voxel dimensions $0.9375 \quad 0.9375 \quad 1.5$ $\mathrm{mm}$ ) were obtained coronally. The caudate nucleus was delineated manually by an expert. This data set was used in previous volumetric and shape analysis studies [4. All the caudate shapes are pre-aligned using rigid transformation.

Global Analysis. Unlike our synthetic data experiment, we do not have enough controls to have non overlapping training and testing data sets. We thus use a leave-one-out method to compute the depth for normal controls, and use the whole control group as the reference to compute the depth for the SPD group. Fig. [4 demonstrates the global differences between normal controls and SPDs, for both left and right caudate. We also use 10000 permutation tests to measure the significant difference of depth for both normal controls and SPD group, resulting in p-values, 0.48 for the left caudate and 0.21 for the right caudate. This indicates based on the global depth-based analysis, both left and right caudates are not significantly different in the SPD and the NC populations.

Local Analysis. For the local shape analysis, we compute the local $\alpha$ values for the median shape of the SPD group, by using the normal control group as the reference shape population. Similar to the permutation test for the synthetic data, the
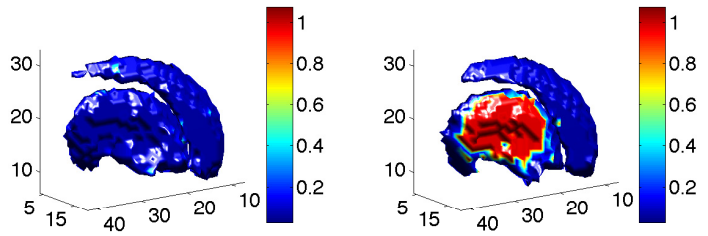

(a) Local measure with $\alpha$ value
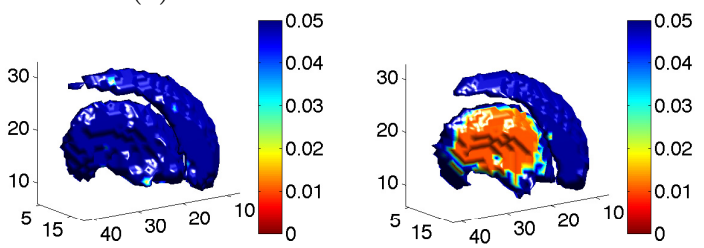

(b) Raw p-values with 10000 permutations
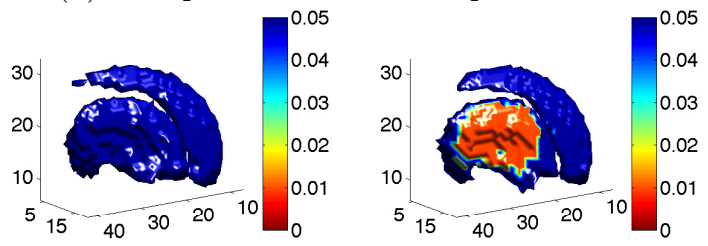

(c) FDR of p-values

Fig. 3. Local analysis on synthetic striatum with $\alpha$ values (a) on normal (left) and abnormal (right) median shapes, and corresponding p-values (b), as well as the FDR of p-values (c)

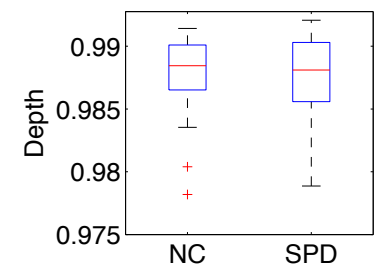

(a) Left caudate

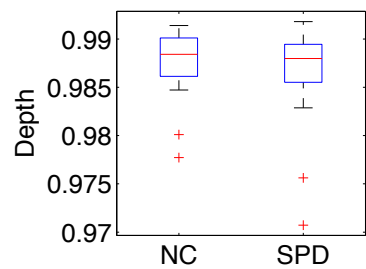

(b) Right caudate
Fig. 4. Band-depth for left and right caudate 


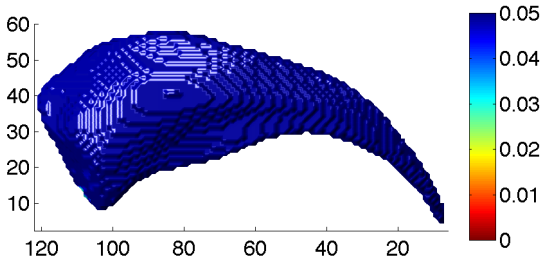

(a) left lateral, p-value

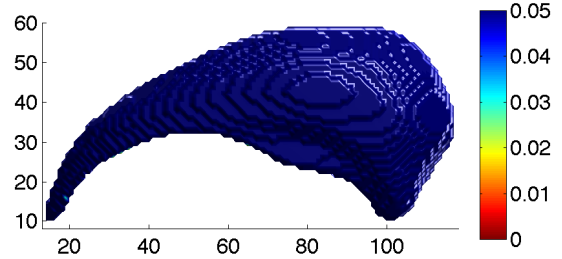

(c) right lateral, p-value

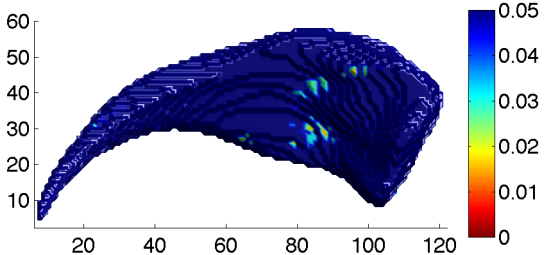

(b) left medial, p-value

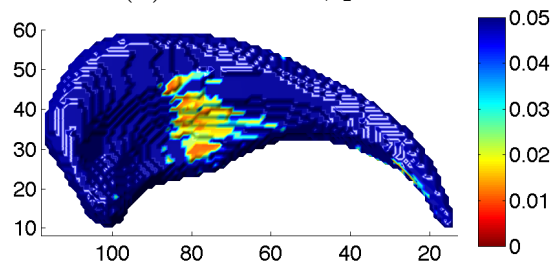

(d) right medial, p-value

Fig. 5. Local p-values. Left caudate SPD median with respect to NC (top), right caudate SPD median with respect to NC (bottom).

p-values with 10000 permutations are shown in Fig. 5. Our method can capture the abnormal region, but based on the local p-values only relatively small regions of the SPD group seem to be significantly different from normal controls.

\section{Discussion and Conclusion}

In this paper we presented a shape analysis framework that can provide both global and local information, yet does not require complex processes to establish point-to-point correspondences. Instead we use the notion of band-depth of functions to order shapes according to how well they "fit in" a shape ensemble. This method allows for the definition of a median and $\alpha$-central regions of a population, which can then be used to compare different population of shapes.

Different from [3], which focuses on augmenting a population atlas with statistical information using weighted band depth, we proposed a fast algorithm to compute the band-depth of shapes represented by binary maps, and most importantly showed how band depth can be used to provide both global and local statistical tests to differentiate between populations. In contrast to other deformation based tools for shape analysis, our approach is non-parametric and naturally captures the probability of a shape belonging to a population. Although it does not provide physical measurements of displacement, these can be computed by deformation or a distance transform to the population median.

Our method was successfully tested with synthetic data, where we were able to clearly separate groups and localize an artificially induced shape change. In addition, our real data experiment supports previous results on shape differences in the caudate of females with SPD, namely, a right sided shape difference in the body of the caudate. 
Acknowledgements This work was supported by NIH grants R01-MH082918, P41EB002025, and R01-HL105241.

\section{References}

1. Davies, R., Twining, C., Taylor, C.: Statistical Models of Shape: Optimisation and Evaluation. Springer, London (2008) 17

2. Gao, Y., Bouix, S.: Synthesis of realistic subcortical anatomy with known surface deformations. In: Levine, J.A., Paulsen, R.R., Zhang, Y. (eds.) MeshMed 2012. LNCS, vol. 7599, pp. 80-88. Springer, Heidelberg (2012) 21$]$

3. Hong, Y., Davis, B., Marron, J.S., Kwitt, R., Niethammer, M.: Weighted functional boxplot with application to statistical atlas construction. In: Mori, K., Sakuma, I., Sato, Y., Barillot, C., Navab, N. (eds.) MICCAI 2013, Part III. LNCS, vol. 8151, pp. 584-591. Springer, Heidelberg (2013) 18,23

4. Levitt, J.J., Styner, M., Niethammer, M., Bouix, S., Koo, M.S., Voglmaier, M.M., Dickey, C.C., Niznikiewicz, M.A., Kikinis, R., McCarley, R.W., Shenton, M.E.: Shape abnormalities of caudate nucleus in schizotypal personality disorder. Schizophrenia Research 110(1-3), 127-139 (2009) 22

5. Loncaric, S.: A survey of shape analysis techniques. Pattern Recognition 31(8), 983-1001 (1998) 17

6. López-Pintado, S., Romo, J.: On the concept of depth for functional data. Journal of the American Statistical Association 104, 718-734 (2009) 18

7. Miller, M.I.: Computational anatomy: Shape, growth, and atrophy comparison via diffeomorphisms. NeuroImage 23, S19-S33 (2004) 17

8. Styner, M., Lieberman, J.A., Pantazis, D., Gerig, G.: Boundary and medial shape analysis of the hippocampus in schizophrenia. Medical Image Analysis 8(3), 197-203 (2004) 17

9. Sun, Y., Genton, M.: Functional boxplots. Journal of Computational and Graphical Statistics 20, 316-334 (2011) 18

10. Sun, Y., Genton, M.G., Nychka, D.W.: Exact fast computation of band depth for large functional datasets: How quickly can one million curves be ranked? Stat. 1, 68-74 (2012) 19

11. Whitaker, R.T., Mirzargar, M., Kirby, R.M.: Contour boxplots: A method for characterizing uncertainty in feature sets from simulation ensembles. IEEE Transactions on Visualization and Computer Graphics 19(12), 2713-2722 (2013) 18 